\title{
ON PARTIALLY ORDERED SETS POSSESSING A UNIQUE ORDER-COMPATIBLE TOPOLOGY
}

\author{
E. S. WOLK ${ }^{1}$
}

1. Introduction. Let $X$ be a partially ordered set (poset) with respect to a relation $\leqq$, and possessing least and greatest elements $O$ and $I$ respectively. Let us call a subset $S$ of $X$ up-directed (downdirected) if and only if for all $x \in S$ and $y \in S$ there exists $z \in S$ such that $z \geqq x, z \geqq y(z \leqq x, z \leqq y)$. Following McShane [2], we call a subset $K$ of $X$ Dedekind-closed if and only if whenever $S$ is an up-directed subset of $K$ and $y=1$.u.b. $(S)$, or $S$ is a down-directed subset of $K$ and $y=$ g.l.b. $(S)$, we have $y \in K$. Let $D$ denote the topology on $X$ whose closed sets are the Dedekind-closed subsets of $X$. Let $g$ denote the well-known interval topology on $X$, which is obtained by taking all sets of the form $[a, b]=\{x \in X \mid a \leqq x \leqq b\}$ as a sub-base for the closed sets. Continuing an investigation which was begun in [5], we shall call a topology $\mathfrak{J}$ on $X$ order-compatible if and only if $\mathfrak{g} \leqq \mathfrak{J}$ $\leqq \mathscr{D} . X$ is said to have a unique order-compatible topology if and only if its $\mathscr{G}$ and $D$ topologies are identical. In [5] we obtained a simple sufficient condition for a poset to possess a unique ordercompatible topology. This result has recently been strengthened by Naito [3]. Let us call a subset $K$ of $X$ diverse if and only if $x \in K$, $y \in K$, and $x \neq y$ imply $x \nless y$. Naito has shown that if a poset $X$ contains no infinite diverse subset, then it possesses a unique order-compatible topology.

The purpose of the present note is to obtain a condition both necessary and sufficient for a poset to have a unique order-compatible topology. However, such a condition has been obtained only for posets satisfying a certain countability restriction (the question of finding a condition which holds in general still remains open). We show that a poset $X$ satisfying such a hypothesis has a unique ordercompatible topology if and only if every diverse subset of $X$ is $g$ closed. With a slight strengthening of our hypothesis we obtain a sufficient condition for this unique topology to be metrizable. We conclude with an investigation of some properties of the order structure of a poset in which every diverse subset is $\mathfrak{g}$-closed.

2. Main results. We first state without proof a lemma which was obtained by Naito [3] and which is an improvement of Lemma 6 of

Received by the editors September 11, 1959.

1 This research was supported by a grant (No. G-5650) from the National Science Foundation. 
[5]. Notation and terminology are the same as in [5]. The obvious dual formulation may be left to the reader.

Lemma 1 (NAIto). Let $X$ be a poset containing no infinite diverse subset, and let $(f(\alpha), \alpha \in A)$ be a net with range $(f)=S \subset X$. Let $y$ be an element of $X$ such that $y$ is the l.u.b. of the range of every subnet of $f$. Then there exists an up-directed set $M \subset S$ such that $y=l . u . b .(M)$.

We now prove our main results.

Theorem 1. Let $X$ be a poset such that the space $(X, g)$ satisfies the first axiom of countability. Then $X$ has a unique order-compatible topology if and only if every diverse subset of $X$ is g-closed. Furthermore, $X$ is a Hausdorff space with respect to this topology.

Proof. A diverse subset of any poset $X$ is always $D$-closed. Hence, if $\mathscr{g}=\mathfrak{D}$, every diverse subset of $X$ is $\mathscr{g}$-closed.

To prove the converse, suppose that every diverse subset of $X$ is $g$-closed, and let $K$ be a D-closed subset. Let $\left\{x_{n}\right\}$ be a sequence of elements of $K$ which converges to an element $y \in X$ in the interval topology. We may assume that $x_{n} \neq y$ for all $n$. We must show that $y \in K$.

We first show that there exists a subsequence of $\left\{x_{n}\right\}$ each member of which is comparable with $y$. For if this is not the case, then $x_{n}$ is incomparable with $y$ for all sufficiently large $n$, and by Lemma 4 of [5] there exists an infinite diverse subset of $X$ which is contained in the range of $\left\{x_{n}\right\}$. But this means that there exists a subsequence $\left\{x_{n_{k}}\right\}$ of $\left\{x_{n}\right\}$ whose range $R$ is diverse. Since by hypothesis $R$ is $g$-closed, and $y \notin R$, we have $\lim x_{n_{k}} \neq y$ in the $g$ topology, which is a contradiction.

Let us assume that each member of the sequence $\left\{x_{n}\right\}$ itself is comparable with $y$. We may assume, furthermore, that $x_{n}<y$ for all $n$ (for if this is not eventually true, then there exists a subsequence $\left\{x_{n_{k}}\right\}$ with $x_{n_{k}}>y$ for all $k$, and the obvious dual proof will apply). Let $S$ be the range of $\left\{x_{n}\right\}$. By the argument of the previous paragraph, $S$ contains no infinite diverse subset. Furthermore, by Lemmas 3 and 5 of [5], $y$ is the l.u.b. of the range of every subnet of $\left\{x_{n}\right\}$. Hence Lemma 1 applies, and we conclude that $S$ contains an up-directed subset $M$ with $y=1$.u.b. $(M)$. Since $M \subset K$ and $K$ is $\mathbb{D}$-closed, we have $y \in K$. Hence $K$ is $\mathscr{g}$-closed, and $\mathscr{g}=\mathbb{D}$.

It remains to show, under the above hypotheses, that the topology $g$ is Hausdorff. Let $\left\{z_{n}\right\}$ be any sequence in $X$ which converges in the $g$ topology to an element $y \in X$. By the arguments of the previous paragraphs we may assume that $z_{n}<y$ for all $n$; and again by Lemmas 
3 and 5 of [5] it follows that $y=$ l.u.b. $\left(z_{n}\right)$. Hence any $g$-convergent sequence of elements of $X$ has a unique limit. Since $(X, \mathfrak{g})$ satisfies the first axiom of countability, this implies that $(X, g)$ is a Hausdorff space.

If $F$ is a closed subset of a topological space $(X, J)$, we shall say that $F$ has a countable system of neighborhoods if and only if there exists a countable family $\mathcal{U} \subset \mathfrak{J}$ such that whenever $F \subset T$ and $T \in \mathfrak{J}$, there is a $U \in \mathcal{U}$ with $F \subset U \subset T$. (Clearly this condition implies the first axiom of countability.)

We now have the following theorem.

THEOREM 2. Let $X$ be a poset in which every closed interval $[a, b]$ has a countable system of neighborhoods in the topology 9 . If $X$ has a unique order-compatible topology, then $X$ is a regular space with respect to this topology.

Proof. It is sufficient to show that if $B$ is a member of a base $B$ for the closed sets of the topology $g$, and $c \notin B$, then there exist disjoint g-open sets $U$ and $V$ such that $B \subset U$ and $c \in V$. (For if $F$ is an arbitrary $g$-closed subset of $X$ and $c \notin F$, then there exists $B \in Q$ with $F \subset B$ and $c \notin B$.) Since the collection of all finite unions of closed intervals of $X$ is a base for the closed sets of the topology $g$, it follows that it is sufficient to show that whenever $[a, b]$ is a closed interval, and $c \notin[a, b]$, then there exist disjoint $g$-open sets $U$ and $V$ with $[a, b] \subset U$ and $c \in V$. Suppose that this is not true for some interval $[a, b]$ in $X$ and some $c \notin[a, b]$. Let $\left\{U_{n} \mid n=1,2, \cdots\right\}$ and $\left\{V_{n} \mid n=1,2, \cdots\right\}$ be decreasing sequences of open sets which form countable neighborhood systems for $[a, b]$ and $c$, respectively. Then $U_{n} \cap V_{m}$ is nonvoid for all $m$ and $n$. For each $n=1,2, \cdots$, choose $x_{n} \in U_{n} \cap V_{n}$. Then the sequence $\left\{x_{n}\right\}$ converges to $c$ in the $\mathscr{g}$ topology and is also eventually in every $g$-open set which contains $[a, b]$. As in the proof of Theorem $1, x_{n}$ must be comparable with $c$ for all sufficiently large $n$ We may therefore again assume that $x_{n}<c$ for all $n$. It then follows, again using Lemmas 3 and 5 of [5], that $c=1$.u.b. $\left(x_{n}\right)$. Furthermore, since $[a, b]$ is closed and $c \notin[a, b]$, there exists $n_{0}$ such that $x_{n} \in[a, b]$ for all $n \geqq n_{0}$. Since $c$ is the unique limit of the sequence $\left\{x_{n}\right\}$, the set $F=\left\{x_{n} \mid n \geqq n_{0}\right\} \cup\{c\}$ is 9 -closed, and also disjoint from $[a, b]$. Let $G=$ complement of $F$. Then $G$ is an $g$-open set containing $[a, b]$ but $\left\{x_{n}\right\}$ is not eventually in $G$, a contradiction.

The question remains open as to whether Theorems 1 and 2 remain valid without some countability assumptions.

It is natural to ask for a purely "order-theoretic" property of $X$ which is sufficient to imply the topological countability hypotheses of 
Theorems 1 and 2. A convenient property to consider is the following.

Property C. There exists a countable subset $R$ of $X$ such that, whenever $J$ and $K$ are disjoint closed intervals in $X$, there exist $r \in R$ and $s \in R$ with $J$ disjoint from $[r, s]$ and $K \subset[r, s]$.

We may now prove

Lemma 2. If a poset $X$ has property $\mathrm{C}$, then (i) $(X, g)$ satisfies the second axiom of countability, and (ii) every closed interval $[a, b]$ in $X$ has a countable system of neighborhoods in the topology $g$.

Proof. (i) follows immediately from the observation that the family of all finite unions of closed intervals of the form $[r, s]$, for $r \in R$ and $s \in R$, is a base for the closed sets of $(X, g)$. To prove (ii), let $B$ be the family of all finite unions of sets $[r, s]$ such that $r \in R$, $s \in R$, and $[r, s]$ is disjoint from $[a, b]$. Then the family of all complements of members of $B$ is a countable neighborhood system for $[a, b]$ in the topology $g$.

Lemma 2, Theorems 1 and 2, and the well-known metrization theorem of Urysohn now imply

THEOREM 3. Let $X$ be a poset with property $\mathrm{C}$ in which every diverse subset is $\mathrm{g}$-closed. Then $X$ has a unique order-compatible topology which is metrizable.

3. Diverse subsets which are closed in the interval topology. It may be of interest also to obtain an "order-theoretic" property which characterizes a poset in which all diverse subsets are $g$-closed. To do this, we shall first give a characterization of the 9 -convergent nets in $X$. Our terminology in regard to nets is that of [1]. In particular, we follow [1] in our use of the terms "eventually" and "frequently."

The following notation will be convenient. If $K \subset X$, we shall write $K^{+}=\{x \in X \mid x \leqq y$ for all $y \in K\}$ and $K^{*}=\{x \in X \mid x \geqq y$ for all $y \in K\}$. If $f$ is a net in $X$, let $\Gamma_{f}$ denote the set of all cofinal subnets of $f$. Then we define

$$
\begin{aligned}
& M_{f}=U\left\{[\operatorname{range}(g)]^{+} \mid g \in \Gamma_{f}\right\}, \\
& N_{f}=U\left\{[\operatorname{range}(g)]^{*} \mid g \in \Gamma_{f}\right\} .
\end{aligned}
$$

Theorem 4. A net $f$ in the poset $X$ converges to an element $y$ in the topology $g$ if and only if $y \in M_{f}^{*} \cap N_{f}^{+}$.

Proof. Suppose that $f g$-converges to $y$ and that $y \in M_{f}^{*} \cap N_{f}^{+}$. Then there exists $m \in M_{f}$ with $y \geq m$, or there exists $n \in N_{f}$ with $y \$ n$. In either case there exists a closed interval $J$ in $X$ such that $J$ 
contains the range of a cofinal subnet of $f$ and $y \notin J$. Then $X-J$ (the complement of $J$ with respect to $X$ ) is an 9 -open neighborhood of $y$, and by hypothesis $f$ is eventually in $X-J$. But this means that $f$ is not frequently in $J$, a contradiction.

To prove the converse, suppose that $f$ does not converge to $y$ in the $g$ topology. Then there exists an $g$-open set $U$ such that $y \in U$ and $f$ is frequently in $X-U$. But $X-U$ is the intersection of a family of members of the usual closed base for the topology $g$. Hence there exists a member $B$ of this closed base such that $y \notin B$ and $f$ is frequently in $B$. But $B$ is of the form $U\left\{J_{i} \mid i=1,2, \cdots, n\right\}$, where each $J_{i}$ is a closed interval; and $f$ is frequently in $B$ implies that $f$ is frequently in some $J_{k}(k=1,2, \cdots, n)$. If $J_{k}=\left[a_{k}, b_{k}\right]$, then $a_{k} \in M_{f}$, $b_{k} \in N_{f}$. But $y \notin J_{k}$, and hence $y a_{k}$ or $y b_{k}$. In either case we have $y \notin M_{f}^{*} \cap N_{f}^{+}$.

Now let $L$ be any infinite diverse subset of $X$. Let $F(L)$ denote the family of all sets of the form $L-F$, where $F$ is a finite subset of $L$. Let us define

$$
A_{L}=\bigcup\left\{K^{+} \mid K \in \mathcal{F}(L)\right\}, \quad B_{L}=\bigcup\left\{K^{*} \mid K \in \mathcal{F}(L)\right\} .
$$

We then have the following theorem.

Theorem 5. An infinite diverse subset $L$ of a poset $X$ is g-closed if and only if $A_{L}^{*} \cap B_{L}^{+} \subset L$.

Proof. Suppose that $L$ is $g$-closed. Let $D=\{(x, K) \mid K \in \mathcal{F}(L)$ and $x \in K\}$. The set $D$ may be up-directed by defining $\left(x_{1}, K_{1}\right) \leqq\left(x_{2}, K_{2}\right)$ if and only if $K_{1} \supset K_{2}$. If we define $f(x, K)=x$, then $f$ is a net on $D$ with values in $L$. We shall show that for this net, $f, M_{f}^{*} \cap N_{f}^{+}=A_{L}^{*} \cap B_{L}^{+}$. Since $\left\{f(x, K) \mid(x, K) \geqq\left(x_{0}, K_{0}\right)\right\}=K_{0}$, it follows that each $K \in \mathcal{F}(L)$ is the range of a cofinal subnet of $f$ (actually a residual subnet). Hence $A_{L} \subset M_{f}, B_{L} \subset N_{f}$. But then $A_{L}^{*} \supset M_{f}^{*}, B_{L}^{+} \supset N_{f}^{+}$, and $A_{L}^{*} \cap B_{L}^{+}$ $\supset M_{f}^{*} \cap N_{f}^{+}$. To prove the reverse inclusion, note that the range of any cofinal subnet $g$ of $f$ obviously contains some $K_{0} \in \mathcal{F}(L)$. Hence [range $(g)]^{+} \subset K_{0^{+}}$, and dually. Then $M_{f} \subset A_{L}, N_{f} \subset B_{L}$, and $A_{L}^{*} \cap B_{L}^{+}$ $\subset M_{f}^{*} \cap N_{f}^{+}$. Hence $A_{L}^{*} \cap B_{L}^{+}=M_{f}^{*} \cap N_{f}^{+}$, and by Theorem 4 we must have $A_{L}^{*} \cap B_{L}^{+} \subset L$.

To prove the converse it is convenient to use the terminology of filters. If $\mathcal{U}$ is a filter on $L$, let us define $P(\mathcal{U})=U\left\{S^{+} \mid S \in \mathcal{U}\right\}, Q(\mathcal{u})$ $=\cup\left\{S^{*} \mid S \in \mathcal{U}\right\}$. Following Ward [4], we say that an element $x$ of $X$ is medial for $\mathcal{U}$ if and only if $x \in[P(\mathcal{U})]^{*} \cap[Q(\mathcal{U})]^{+}$. Ward [4] has shown that $x$ is medial for an ultrafilter $\mathfrak{u}$ on $X$ if and only if $\mathfrak{u}$ is 9 -convergent to $x$.

Now to show that $L$ is $g$-closed it is sufficient to show that if $\mathcal{U}$ is 
any ultrafilter on $L$, then $\mathcal{U}$ does not converge to any point of $X-L$. We distinguish two cases. First, suppose that there exists $y \in L$ with $y \in S$ for all $S \in \mathcal{U}$. Then we must have $\{y\} \in \mathcal{u}$, and $\mathcal{u}$ cannot $g$ converge to any point of $X$ other than $y$ (since $(X, \mathfrak{g})$ is a $T_{1}$-space). Suppose then that there exists no $y \in L$ with $y \in S$ for all $S \in \mathcal{U}$. Then $\mathcal{U}$ must contain the filter $\mathscr{F}=\mathscr{F}(L)$. It is easy to verify that $\mathcal{U} \supset \mathcal{F}$ implies that $[P(\mathcal{U})]^{*} \cap[Q(\mathcal{U})]^{+} \subset[P(\mathcal{F})]^{*} \cap[Q(\mathcal{F})]^{+}=A_{L}^{*} \cap B_{L}^{+}$. But by hypothesis $A_{L}^{*} \cap B_{L}^{+} \subset L$. Thus $x \in L$ whenever $x$ is medial for $\mathcal{U}$, which completes the proof.

THEOREM 6. Every diverse subset of a poset $X$ is 9 -closed if and only if $A_{L}^{*} \cap B_{L}^{+}$is empty for every infinite diverse subset $L$.

Proof. Suppose that every diverse subset of $X$ is 9 -closed, and that for some infinite diverse $L$ there is an element $z \in A_{L}^{*} \cap B_{L}^{+}$. By Theorem 5, $z \in L$. Let $H=L-\{z\}$. Since $\mathcal{F}(H) \subset \mathcal{F}(L)$, we have $A_{H} \subset A_{L}, B_{H} \subset B_{L}$. Hence $A_{H}^{*} \supset A_{L}^{*}, B_{H}^{+} \supset B_{L}^{+}$, and $z \in A_{H}^{*} \cap B_{H}^{+}$. But $H$ is $\mathrm{g}$-closed and $z \notin H$, contradicting Theorem 5 . The converse proposition follows trivially from Theorem 5 .

Theorem 6 suggests that it is slightly "pathological" for a poset to contain infinite diverse subsets all of which are 9 -closed. However, the following is a simple example of such a poset. Consider the following sets of points in the complex plane. Let $X_{1}=\{(1 / n, 0) \mid n=1,2, \cdots\}$, $X_{2}=\{(0,1 / n) \mid n= \pm 1, \pm 2, \cdots\}$. Let $X=X_{1} \cup X_{2}$. We partially order $X$ as follows. Define $(0,1 / m) \leqq(0,1 / n)$ if and only if $1 / m \leqq 1 / n$ in the usual ordering. For $n>0$, define $(1 / m, 0)<(0,1 / n)$ if and only if $1 / m \leqq 1 / n$. For $n<0$, define $(1 / m, 0)>(0,1 / n)$ if and only if $1 / m \leqq 1 / n$. The set $X_{1}$ is diverse. With this ordering it is clear that $X$ satisfies the hypotheses of Theorem 3 .

\section{REFERENCES}

1. J. L. Kelley, General topology, Van Nostrand, 1955.

2. E. J. McShane, Order-preserving maps and integration processes, Annals of Mathematics Studies, vol. 31, Princeton, 1953.

3. T. Naito, On a problem of Wolk in interval topologies, Proc. Amer. Math. Soc. vol. 11 (1960) pp. 156-158.

4. A. J. Ward, On relations between certain intrinsic topologies in partially ordered sets, Proc. Cambridge Philos. Soc. vol. 51 (1955) pp. 254-261.

5. E. S. Wolk, Order-compatible topologies on a partially ordered set, Proc. Amer. Math. Soc. vol. 9 (1958) pp. 524-529.

UNIVERSITY OF CONNECTICUT 